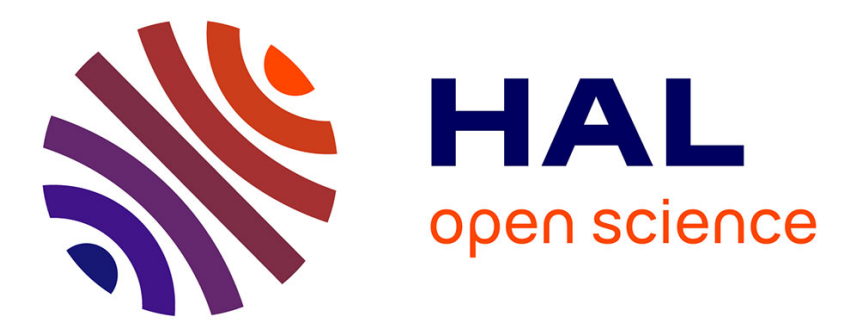

\title{
A multivariate multifractal analysis of lacunary wavelet series
}

\author{
Stéphane Jaffard, Stéphane Seuret, Herwig Wendt, Patrice Abry, Roberto \\ Leonarduzzi
}

\section{- To cite this version:}

Stéphane Jaffard, Stéphane Seuret, Herwig Wendt, Patrice Abry, Roberto Leonarduzzi. A multivariate multifractal analysis of lacunary wavelet series. IEEE 8th International Workshop on Computational Advances in Multi-Sensor Adaptive Processing (CAMSAP 2019), Dec 2019, Guadeloupe, France. 10.1109/CAMSAP45676.2019.9022481 . hal-03034146

\section{HAL Id: hal-03034146 \\ https://cnrs.hal.science/hal-03034146}

Submitted on 1 Dec 2020

HAL is a multi-disciplinary open access archive for the deposit and dissemination of scientific research documents, whether they are published or not. The documents may come from teaching and research institutions in France or abroad, or from public or private research centers.
L'archive ouverte pluridisciplinaire HAL, est destinée au dépôt et à la diffusion de documents scientifiques de niveau recherche, publiés ou non, émanant des établissements d'enseignement et de recherche français ou étrangers, des laboratoires publics ou privés. 


\section{A multivariate multifractal analysis of lacunary wavelet series}

\author{
Stéphane Jaffard, Stéphane Seuret \\ LAMA, Univ. Paris-Est, \\ UPEM, UPEC, CNRS, Créteil, France. \\ firstname. lastnamedu-pec.fr
}

\author{
Herwig Wendt \\ IRIT, Univ. Toulouse, \\ CNRS, Toulouse, France. \\ herwig.wendteirit.fr
}

\author{
Patrice Abry, Roberto Leonarduzzi \\ Univ Lyon, Ens de Lyon, Univ Claude Bernard, \\ CNRS, Laboratoire de Physique, Lyon, France. \\ firstname. lastnamedens-lyon. fr
}

\begin{abstract}
Multivariate multifractal analysis proposes to estimate the multivariate multifractal spectrum of several signals as a way to reveal the correlations between their singularity sets. Recently discovered failures of the "natural" multifractal formalism challenge its foundations, and raise questions on the information it provides. We investigate these questions by supplying some general results on the multivariate multifractal analysis of processes satisfying the large intersection property, and illustrate it on random lacunary wavelet series.
\end{abstract}

\section{INTRODUCTION}

Context: Multifractal analysis. The purpose of multifractal analysis is to analyze functions or measures (random or deterministic) by estimating the size of the sets of points where they have a given pointwise regularity. Mathematically, this information is encapsulated through the multifractal spectrum $\mathcal{D}(H)$, which yields the Hausdorff dimensions of the sets $E(H)$ where a pointwise regularity exponent takes the value $H$ [1]. In practice, for a signal or an image, a direct estimation of $\mathcal{D}(H)$ is unfeasible; the purpose of multifractal formalisms is to estimate it through the computation of a Legendre spectrum which, for univariate data, yields an upper bound of $\mathcal{D}(H)$ [2], and coincides with it for important classes of models (cf. e.g., [3] and ref. therein). Thus, the derivation of practically computable Legendre spectra as close as possible to $\mathcal{D}(H)$ is a central issue.

Related works. Recent applications involve simultaneous recordings of large collections of signals or images, thus calling for a multivariate multifractal analysis. When several functions $X_{1}(x), \cdots, X_{M}(x)$ are involved, the natural mathematical quantity considered is the multivariate multifractal spectrum $\mathcal{D}\left(H_{1}, \cdots H_{M}\right)$, which yields the Hausdorff dimensions of the sets of points where the regularity exponent of each signal $X_{i}$ takes a given value $H_{i}$. A natural multivariate multifractal formalism was proposed in [4], initially in the context of turbulence, and has been used in a wide range of applications. However, its validity was recently questioned, and a dramatic turning point was the discovery that, in general, this formalism does not yield an upper bound for the multivariate spectrum [5]; counterexamples are supplied by classical models, such as independent Lévy processes, or couples of anti-correlated binomial cascades. This unexpected failure raises the problems of understanding under which conditions the formalism is valid (i.e. yields an upper bound) and which information it supplies when such conditions are not fulfilled (or cannot be verified), in particular what it reveals about the correlations between the locations of the singularities of the components $X_{i}$.

Goals, contributions and outline. We investigate these questions following the guideline supplied by a simple stochastic model: Lacunary wavelet series (LWS). Their sample paths were shown to be multifractal [6], and we study couples of such processes, either independent or with tuned correlations. In particular, we show that, in this case too, the natural multivariate formalism fails. This confirms a major source of failure when the singularity sets of the processes satisfy the large intersection property; we back this intuition by general mathematical results on multivariate processes which share this property. In Section III we recall the basic notions in multivariate multifractal analysis. In Section III we derive mathematical results on the bivariate multifractal analysis of processes satisfying the large intersection property. In Section IV we study, theoretically and numerically, how the bivariate spectrum of LWS changes when their correlation is tuned.

\section{Multivariate multifRactal analysis}

For simplicity, in the following, we only consider the bivariate case $(M=2)$ where functions are defined on $\mathbb{R}$. Higher dimensional extensions easily follow.

\section{A. Multifractal spectrum}

Multifractal analysis quantifies the fluctuations of the regularity of a signal $X(t)$. Pointwise regularity of functions is usually measured using the Hölder exponent, $h(t)$, defined as follows: $X \in C^{\alpha}(t), \alpha \geq 0$, if there exist a polynomial $P_{t}$ with $\operatorname{deg}\left(P_{t}\right)<\alpha$ and a constant $C>0$ such that

$$
\left|X(t+a)-P_{t}(t+a)\right| \leq C|a|^{\alpha},|a| \rightarrow 0,
$$

$h(t)$ is the largest $\alpha$ such that $(1)$ is satisfied,

$$
h(t) \triangleq \sup \left\{\alpha: X \in C^{\alpha}(t)\right\} \geq 0,
$$

see e.g. [7] for alternative notions of pointwise regularity.

Let $\mathbf{h}(t) \triangleq\left(h_{1}(t), h_{2}(t)\right)$ denote the Hölder exponents of the components of the bivariate signal $\mathbf{X}=\left(X_{1}, X_{2}\right)$. Let $\mathbf{H}=\left(H_{1}, H_{2}\right)$; the bivariate multifractal spectrum $\mathcal{D}\left(H_{1}, H_{2}\right)$ of $X$ is defined as (see e. g. [4], [8])

$$
\mathcal{D}(\mathbf{H}) \triangleq \operatorname{dim}_{H}\left(E_{\mathbf{H}}\right), \text { where } E_{\mathbf{H}}=\{t: \mathbf{h}(t)=\mathbf{H}\}
$$


and $\operatorname{dim}_{H}$ denotes the Hausdorff dimension (by convention $\left.\operatorname{dim}_{H}(\emptyset)=-\infty\right)$. It provides a global, geometric description of the pointwise regularity properties of the components $X_{i}$; here and in the following the subscript $i$ refers to the $i$-th component $(i=1,2)$. Its precise shape and width quantify key information regarding correlations between the exponents $h_{i}(t)$ [5], [9], [10].

\section{B. Wavelet leader bivariate multifractal formalism}

The estimation of the multifractal spectrum requires numerically robust formulas: multifractal formalisms. A univariate formalism constructed from the multiscale statistics of wavelet leaders [2], [11], has recently been extended to the multivariate setting, see e.g. [5], [8]-[10], [12], and is recalled now.

Let $N>0$, and let $\psi$ denote a "mother wavelet", i.e. an oscillating well-localized function in $C^{N}$, such that $\left\{\psi_{j, k}(t)=\right.$ $\left.2^{-j / 2} \psi\left(2^{-j} t-k\right)\right\}_{(j, k) \in \mathbb{Z}^{2}}$ forms an orthonormal basis of $\mathcal{L}^{2}(\mathbb{R})$. The wavelet coefficients of a univariate function $f$ are $d_{f}(j, k)=2^{-j / 2}\left\langle\psi_{j, k} \mid f\right\rangle$. The wavelet leaders of $f$ are $\ell_{f}(j, k) \triangleq \sup _{\lambda^{\prime} \subset 3 \lambda_{j, k}}\left|d_{f}\left(\lambda^{\prime}\right)\right|$, where $\lambda_{j, k}=\left[k 2^{j},(k+1) 2^{j}\right)$ denotes the dyadic interval of size $2^{j}$ and $3 \lambda_{j, k}$ stands for the union of $\lambda_{j, k}$ with its two closest neighbors [2], [11]. In the bivariate case, the structure functions $S\left(q_{1}, q_{2}, j\right)$ and scaling function $\zeta\left(q_{1}, q_{2}\right)$ of $\mathbf{X}=\left(X_{1}, X_{2}\right)$ are defined by

$$
S\left(q_{1}, q_{2}, j\right) \triangleq 2^{j} \sum_{k} L_{X_{1}}(j, k)^{q_{1}} L_{X_{2}}(j, k)^{q_{2}} \sim c_{q} 2^{j \zeta\left(q_{1}, q_{2}\right)}
$$

(for $j \rightarrow-\infty$ ), where the order $q_{i}$ of empirical moments is taken in some interval including zero. In contradistinction with the univariate case, the bivariate Legendre spectrum

$$
\mathcal{L}(\mathbf{H})=\inf _{\mathbf{q}}(1+\langle\mathbf{q}, \mathbf{H}\rangle-\zeta(\mathbf{q})), \quad \mathbf{q}=\left(q_{1}, q_{2}\right),
$$

does not always yield an upper bound for $\mathcal{D}(\mathbf{H})$, see [5] for a discussion of conditions under which it is the case.

The case where $X_{1}$ and $X_{2}$ are independent was studied in [5]: Under a stationarity hypothesis, Legendre spectra satisfy

$$
\mathcal{L}\left(H_{1}, H_{2}\right)=\mathcal{L}_{1}\left(H_{1}\right)+\mathcal{L}_{2}\left(H_{2}\right)-1 .
$$

This formula is backed by the remark that, applied to multifractal spectra, the corresponding formula

$$
\mathcal{D}\left(H_{1}, H_{2}\right)=\mathcal{D}_{1}\left(H_{1}\right)+\mathcal{D}_{2}\left(H_{2}\right)-1
$$

follows the generic rule that, for smooth sets, codimensions of intersections of sets add up [13] (note that, if 77) yields a negative dimension it is understood that the corresponding set is empty). However, a different formula applies to models for which the lower singularity sets $E \leq(H)=\{x \in \mathbb{R}: h(x) \leq$ $H\}$ are sets with large intersections, i.e. any couple among them satisfies $\operatorname{dim}_{H}(A \cap B)=\min \left(\operatorname{dim}_{H} A, \operatorname{dim}_{H} B\right)$, see [14], [15]. Though the $E_{H}$ are not sets with large intersection, this leads to the alternative conjectured formula for the bivariate spectrum of independent processes of this type:

$$
\mathcal{D}\left(H_{1}, H_{2}\right)=\min \left(\mathcal{D}_{1}\left(H_{1}\right), \mathcal{D}_{2}\left(H_{2}\right)\right)
$$

\section{LARGE INTERSECTION PROCESSES}

For several standard models such as Lévy processes, lacunary and random wavelet series, the sets $E \leq(H)$ are sets of large intersection, see [16]-[18] and ref. therein. The reason is that these sets are obtained as limsup of intervals centered at equidistributed points; as a consequence the following property holds: For $s=\operatorname{dim}_{H}\left(E^{\leq}(H)\right)$, they belong to the $\mathcal{G}^{s}$ class of Falconer, which is composed of sets $A$ which are $G_{\delta}$ sets (i.e. countable intersections of open sets) and such that, for any countable sequence of similarity transformations $f_{i}$, $\operatorname{dim}_{H}\left(\cap f_{i}(A)\right) \geq s$. An important consequence is that there exists a $s$-dimensional Hausdorff measure of $A$ which is positive, see [14], [15]. We now investigate which implications on bivariate spectra hold in this setting.

We consider a couple of functions $\left(X_{1}, X_{2}\right)$, deterministic or random, without assumptions on their correlations in the random case. We assume that there exists an interval $\left[H^{-}, H^{+}\right]$such that: $\forall H \in\left[H^{-}, H^{+}\right]$, the set $E^{\leq}(H)$ belongs to $\mathcal{G}^{s}$ with $s=\mathcal{D} \leq(H)$ (in the examples we mentioned, the range $\left[H^{-}, H^{+}\right]$coincides with $\left[H_{\min }, H_{\max }\right]$ of all Hölder exponents taken by the process). It follows that (in this range of exponents) the bivariate lower spectrum $\mathcal{D} \leq\left(H_{1}, H_{2}\right) \triangleq$ $\operatorname{dim}_{H}\left(\left\{t: h_{1}(t) \leq H_{1}\right.\right.$ and $\left.\left.h_{2}(t) \leq H_{2}\right\}\right)$ satisfies

$$
\begin{aligned}
\mathcal{D} \leq\left(H_{1}, H_{2}\right) & =\operatorname{dim}_{H}\left(E_{1}^{\leq}\left(H_{1}\right) \cap E_{2}^{\leq}\left(H_{2}\right)\right) \\
& =\min \left(\mathcal{D}_{1}^{\leq}\left(H_{1}\right), \mathcal{D}_{2}^{\leq}\left(H_{2}\right)\right) .
\end{aligned}
$$

In particular, it can not yield information on correlations between the two processes. On the opposite, determining the bivariate spectrum $\mathcal{D}\left(H_{1}, H_{2}\right)$ requires information on these correlations. However, we will see that, under an additional assumption, a part of this spectrum does not depend on these correlations. We now assume that, for each process $X_{i}$ $(i=1,2)$ the function $H \rightarrow \mathcal{D}_{i}^{\leq}(H)$ is continuous and strictly increasing on $\left[\mathrm{H}_{i}^{-}, \mathrm{H}_{i}^{+}\right]$(these assumptions are verified by the examples we mentioned). The corresponding process will be called a large intersection process. It follows that, for $i=1,2$, $\mathcal{D}_{i}(H)=\mathcal{D}_{i}^{\leq}(H)$. We define the ridge of the bivariate spectrum as the set: $\left\{\left(H_{1}, H_{2}\right): \mathcal{D}_{1}^{\leq}\left(H_{1}\right)=\mathcal{D}_{2}^{\leq}\left(H_{2}\right)\right\}$. Note that, if the two processes share the same law, then the ridge simply is a segment of the diagonal $H_{1}=H_{2}$.

Theorem 1: Under the previous assumptions, the bivariate spectrum of $\left(X_{1}, X_{2}\right)$ satisfies that, for any $\left(H_{1}, H_{2}\right)$ which belongs to the ridge, $\mathcal{D}\left(H_{1}, H_{2}\right)=\mathcal{D}_{1}\left(H_{1}\right)\left(=\mathcal{D}_{2}\left(H_{2}\right)\right)$.

Proof: The upper bound is straightforward since $E\left(H_{1}, H_{2}\right)$ is included in $E_{1}\left(H_{1}\right)$. Conversely, the $\mathcal{G}^{s}$ class assumption implies that $E \leq\left(H_{1}, H_{2}\right)$ has a positive $\mathcal{D} \leq\left(H_{1}, H_{2}\right)$ dimensional Hausdorff measure; $E\left(H_{1}, H_{2}\right)$ contains

$$
E^{\leq}\left(H_{1}, H_{2}\right)-\bigcup_{H<H_{1}} E^{\leq}\left(H, H_{2}\right)-\bigcup_{H<H_{2}} E^{\leq}\left(H_{1}, H\right) .
$$

By the increasing spectrum assumption, for $H<H_{1}$ (resp. $\left.H<H_{2}\right)$, the sets $E^{\leq}\left(H, H_{2}\right)$ and $E^{\leq}\left(H_{1}, H\right)$ have a vanishing $\mathcal{D} \leq\left(H_{1}, H_{2}\right)$-dimensional Hausdorff measure; since the unions are countable, $E\left(H_{1}, H_{2}\right)$ has a positive $\mathcal{D} \leq\left(H_{1}, H_{2}\right)$ dimensional Hausdorff measure; hence the lower bound holds. 
Remark: The support of the bivariate spectrum can boil down to the ridge, for instance when the functions $X_{1}$ and $X_{2}$ coincide. Thus the determination of the bivariate spectrum outside of the ridge necessarily requires information on the correlation between the two processes.

\section{LACUNARY WAVELET SERIES}

We start by recalling the model studied in [6]. For the sake of simplicity, the random process $X$ is constructed on $[0,1]$. Let $\eta \in(0,1)$ and $\alpha>0$. At scale $j \leq 0,\left[2^{-\eta j}\right]$ dyadic intervals $(\lambda \subset[0,1])$ are drawn at random, and for these intervals, $c_{\lambda}^{1}=2^{\alpha j}$; the other wavelet coefficients are set to 0 . Additionally, the coefficients are independent at each scale. The parameters of the series are its lacunarity $\eta$ and its uniform regularity $H_{\min }=\alpha$. The multifractal spectrum of $X$ is

$$
\begin{aligned}
\mathcal{D}(H) & =\eta H / \alpha & & \text { if } H \in[\alpha, \alpha / \eta] \\
& =-\infty & & \text { else. }
\end{aligned}
$$

We now consider two LWS $X_{1}, X_{2}$ of parameters $\left(\eta_{1}, \alpha_{1}\right)$ and $\left(\eta_{2}, \alpha_{2}\right)$. The results of Section III apply: Without any correlation assumption, $\forall\left(H_{1}, H_{2}\right) \in\left[\alpha_{1}, \alpha_{1} / \eta_{1}\right] \times\left[\alpha_{2}, \alpha_{2} / \eta_{2}\right]$,

$$
\mathcal{D} \leq\left(H_{1}, H_{2}\right)=\min \left(\frac{\eta_{1} H_{1}}{\alpha_{1}}, \frac{\eta_{2} H_{2}}{\alpha_{2}}\right) .
$$

The ridge is included inside the line $\frac{\eta_{1} H_{1}}{\alpha_{1}}=\frac{\eta_{2} H_{2}}{\alpha_{2}}$ and, on the ridge, $\mathcal{D}\left(H_{1}, H_{2}\right)$ is given by 9 .

Additionally, since the sets $E_{1}\left(H_{1}\right)$ and $E_{2}\left(H_{2}\right)$ are empty if $H_{1}<\alpha_{1}$ or if $H_{2}<\alpha_{2}$, it follows that $E \leq\left(\alpha_{1}, \alpha_{2}\right)=$ $E\left(\alpha_{1}, \alpha_{2}\right)$, so that $\mathcal{D}\left(\alpha_{1}, \alpha_{2}\right)=\mathcal{D} \leq\left(\alpha_{1}, \alpha_{2}\right)$ (note that $\left(\alpha_{1}, \alpha_{2}\right)$ is not in the ridge except if $\left.\eta_{1}=\eta_{2}\right)$.

1) Independent LWS: If $X_{1}$ and $X_{2}$ are independent, then the assumptions under which the derivation of (6) is performed in [5] are verified, so that, if $\left(H_{1}, H_{2}\right) \in\left[\alpha_{1}, \alpha_{1} / \eta_{1}\right] \times$ $\left[\alpha_{2}, \alpha_{2} / \eta_{2}\right]$, then

$$
\mathcal{L}\left(H_{1}, H_{2}\right)=\frac{\eta_{1} H_{1}}{\alpha_{1}}+\frac{\eta_{2} H_{2}}{\alpha_{2}}-1
$$

(and $\mathcal{L}\left(H_{1}, H_{2}\right)=-\infty$ else). Therefore $\mathcal{L}\left(H_{1}, H_{2}\right)$ does not supply an upper bound for $\mathcal{D}\left(H_{1}, H_{2}\right)$, see Fig. 1) (c).

2) Correlated lacunary wavelet series: We consider a first LWS $X_{1}$ of parameters $(\alpha, \eta)$, and construct a second random process $X_{2}$, correlated with $X_{1}$ : We pick $\omega>\eta$ and $\beta>$ 0 . For each interval $\lambda$ picked in the construction of $X_{1}$, we consider the unique dyadic interval $\tilde{\lambda}$ of scale $\tilde{j}=[\omega j]$ ( where $j$ is the scale of $\lambda$ ) which contains $\lambda$; then we set $c_{\tilde{\lambda}}^{2}=2^{\beta \tilde{j}}$, and all other wavelet coefficients of $X_{2}$ are set to 0 . Considered independently of $X_{1}, X_{2}$ is another lacunary wavelet series of lacunarity parameter $\tilde{\eta}=\eta / \omega$ and uniform regularity $\beta$ (note that, strictly speaking, this is not true because the number of nonvanishing wavelet coefficients at a given scale is random and may slightly differ from $\left[2^{\tilde{\eta} j}\right]$ but, clearly, this does not alter the conclusions concerning the spectra).

Remark: The case $\omega=1$ and $\alpha=\beta$ corresponds to the case where $X_{1}=X_{2}$. The case $\omega=1$ and $\alpha \neq \beta$ corresponds to the case where $X_{1}$ is analyzed jointly with its fractional primitive of order $\beta-\alpha$.
We now recall how the pointwise regularity of $X_{1}$ is derived in [6]. Let $\Lambda_{1}$ denote the random collection of dyadic intervals indexing nonvanishing wavelet coefficients of $X_{1}$, and $\Lambda_{2}$ denote those of $X_{2}$. Let $\theta \in[\eta, 1]$; we consider "dilated" intervals constructed as follows: $\lambda_{\theta}$ has the same center as $\lambda \in \Lambda_{1}$, and width $2^{\theta j}$, and we denote by $F_{\theta}$ the limsup of the $\lambda_{\theta}$ (i.e. the set of points that belong to an infinite number of the $\lambda_{\theta}$ for $\lambda \in \Lambda_{1}$ ). The key result that allows to derive the multifractal spectrum is that, if $x \in F_{\theta}$, then $h_{1}(x) \leq \alpha / \theta$ and if $x \notin F_{\theta}$, then $h_{1}(x) \geq \alpha / \theta$. It follows that

$$
x \in G_{\theta}:=\bigcap_{\theta^{\prime}<\theta} F_{\theta^{\prime}}-\bigcup_{\theta^{\prime}>\theta} F_{\theta^{\prime}} \Longleftrightarrow h_{1}(x)=\alpha / \theta,
$$

and the large intersection process assumption implies that, if $\theta \in[\eta, 1]$, then $\operatorname{dim}\left(G_{\theta}\right)=\eta / \theta$.

In order to derive the bivariate spectrum of $X_{1}$ and $X_{2}$, we separate two cases:

- Let $\theta \in[\omega, 1]$. If $x \in F_{\theta}$, then we already know that $h_{1}(x) \leq \alpha / \theta$, and $x$ belongs to an infinite number of the $\tilde{\lambda}$ (or one of its two closest neighbors), so that $h_{2}(x)=\beta$.

- Let $\theta \in[\eta, \omega]$. If $x \in F_{\theta}$, then as before, $h_{1}(x) \leq \alpha / \theta$, and $x$ belongs to an infinite number of the $\lambda_{\theta}$, which can be seen as an interval of same center as $\tilde{\lambda}$ and of width $|\tilde{\lambda}|^{\theta / \omega}$ so that $h_{2}(x) \leq \beta \omega / \theta$.

Lower bounds follow in the same way, and we obtain that the regularity at $x$ of both series is determined by the $G_{\theta}$ to which $x$ belongs. For a given $\theta \in[\omega, 1]$, the dimension of the corresponding set is $\eta / \theta$. The following result follows, where the spectrum is parametrized as a function of $H_{1}$.

Theorem 2: The bivariate multifractal spectrum of two correlated lacunary wavelet series of parameters $(\alpha, \eta, \omega)$ is supported by the two following (not colinear) straight lines:

$$
\begin{gathered}
\text { if } H_{1} \in\left[\alpha, \frac{\alpha}{\omega}\right], \quad H_{2}=\beta \text { and } \mathcal{D}\left(H_{1}, H_{2}\right)=H_{1} \frac{\eta}{\alpha} \\
\text { if } H_{1} \in\left[\frac{\alpha}{\omega}, \frac{\alpha}{\eta}\right], H_{2}=H_{1} \frac{\beta \omega}{\alpha} \text { and } \mathcal{D}\left(H_{1}, H_{2}\right)=H_{1} \frac{\eta}{\alpha} .
\end{gathered}
$$

\section{NUMERICAL ILLUSTRATIONS}

Monte Carlo simulation. We illustrate the above theoretical results using 100 realizations of independent LWS of sample size $2^{16}$, for two different sets of parameters: $\left(\alpha_{1}, \eta_{1}\right)=(0.6,0.85)$ and $\left(\alpha_{2}, \eta_{2}\right)=(0.6,0.85)$ for $X_{1}$ and $X_{2}$, respectively, corresponding to a multifractal spectrum with relatively narrow support, and $\left(\alpha_{1}, \eta_{1}\right)=(0.5,0.4)$ and $\left(\alpha_{2}, \eta_{2}\right)=(0.4,0.4)$ for $X_{1}$ and $X_{2}$, respectively, which corresponds to a multifractal spectrum with larger support and marginal spectra that do not coincide. Sample paths and increments for the two parameter settings are plotted in Fig1. 11 (a). Estimation is performed by linear regression of $\log _{2} S\left(q_{1}, q_{2}, j\right)$ for scales $j$ from -10 to -6 and -7 to -4 for the two parameter sets, respectively, for a sufficiently wide range of positive and negative moments $\left(q_{1}, q_{2}\right)$ so as to cover the theoretical support of the multifractal spectra. Numerical process synthesis and estimation procedures have been implemented by ourselves and are available upon request. 

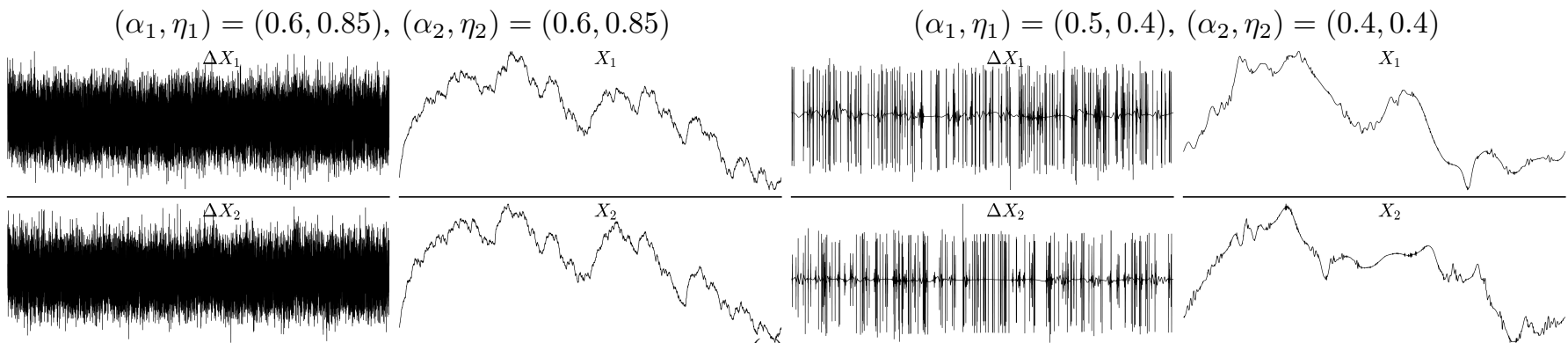

(a)
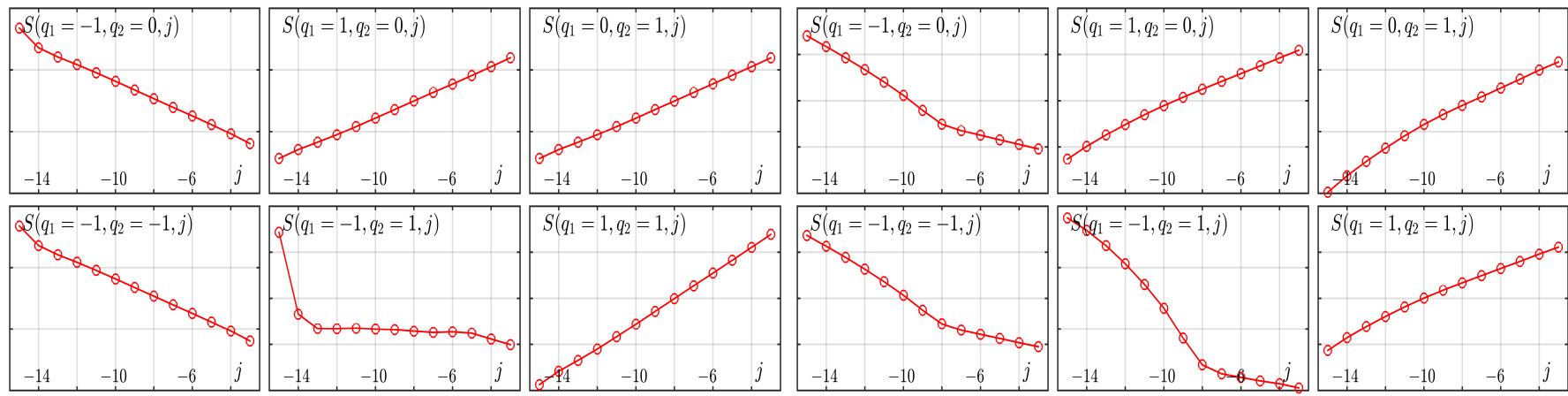

(b)
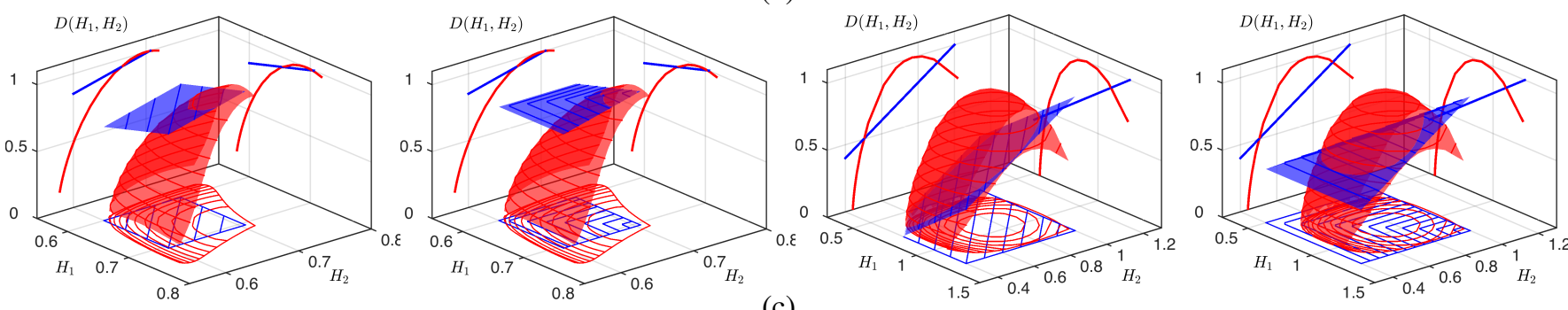

Fig. 1. Increments and sample paths (a), and structure functions (b) for bivariate LWS for two different parameter settings, and estimated (red) vs. theoretical (blue) multifractal spectra (c); the theoretical spectra (in blue) plotted in (c.1) and (c.3) correspond to expression (10) for the Legendre spectrum, those in (c.2) and (c.4) correspond to the expression 9] for the multifractal spectrum.

Structure functions. Log-log plots of average structure functions $S\left(q_{1}, q_{2}, j\right)$ for several values of $\left(q_{1}, q_{2}\right)$ are plotted as a function of scale $j$ in Fig. 1 (b). They show that the power laws postulated in Eq. (4) hold for a large range of scales, and in particular for the range over which linear regressions are performed for the estimation of $\zeta\left(q_{1}, q_{2}\right)$ and $\mathcal{L}(\mathbf{H})$.

Theoretical spectra and Legendre spectra. Fig. 1 (c) plots averages of Legendre spectra estimates (5) (red surfaces and lines) together with theoretical spectra, once corresponding to the theoretical expression (10) for the Legendre spectrum (left columns) and once to that for the multifractal spectrum (9) (right columns), respectively. Clearly, the predictions for the Legendre spectrum and theoretical bivariate multifractal spectrum do not coincide, while the marginal spectra (solid lines) do. Moreover, the estimated Legendre spectra provide a better upper bound for expression (10) than for expression (9), which further corroborates the above theoretical results. The fact that the estimated and theoretical Legendre spectra do not match perfectly is not surprising since it is well documented that numerical estimation for processes with lacunarity is notoriously difficult.

\section{CONCLUSION}

The present study confirms the failure of the multifractal formalism for couples of processes which satisfy the large intersection property. In this case, the Legendre spectrum does not bound the multifractal spectrum, and fails to provide information describing the cross-correlations between components, which was its original goal. Indeed, it is shown that a part of the bivariate spectrum is independent of the correlations between the components. These theoretical results are confirmed by numerical results obtained on sample paths of LWS. This raises two important questions: How could a non-trivial upper bound of the multifractal spectrum be recovered in practice? and which information is supplied by the bivariate Legendre spectrum? The example of correlated LWS yields new insights on the key problem of understanding how the correlations of several processes are reflected in their multivariate spectrum.

\section{ACKNOWLEDGMENT}

Work supported by ANR-16-CE33-0020 MultiFracs, France. 


\section{REFERENCES}

[1] G. Parisi and U. Frisch, "Fully developed turbulence and intermittency," in Turbulence and Predictability in geophysical Fluid Dynamics and Climate Dynamics, ser. Proc. of Int. School, M. Ghil, R. Benzi, and G. Parisi, Eds. Amsterdam: North-Holland, 1985, p. 84.

[2] S. Jaffard, "Wavelet techniques in multifractal analysis," in Fractal Geometry and Applications: A Jubilee of Benoît Mandelbrot, M. Lapidus and M. van Frankenhuijsen, Eds., Proc. Symposia in Pure Mathematics, vol. 72(2). AMS, 2004, pp. 91-152.

[3] P. Abry, S. Jaffard, and H. Wendt, "Irregularities and scaling in signal and image processing: Multifractal analysis," Benoit Mandelbrot: A Life in Many Dimensions, M. Frame and N. Cohen, Eds., World scientific publishing, pp. 31-116, 2015.

[4] C. Meneveau, K. Sreenivasan, P. Kailasnath, and M. Fan, "Joint multifractal measures - theory and applications to turbulence," Phys. Rev. A, vol. 41, no. 2, pp. 894-913, 1990.

[5] S. Jaffard, S. Seuret, H. Wendt, R. Leonarduzzi, and P. Abry, "Multifractal formalisms for multivariate analysis," Proc. Royal Society A, 2019.

[6] S. Jaffard, "On lacunary wavelet series," Annals of Applied Probability, vol. 10, no. 1, pp. 313-329, 2000.

[7] P. Abry, S. Jaffard, R. Leonarduzzi, C. Melot, and H. Wendt, New exponents for pointwise singularity classification, 2017.

[8] S. Jaffard, S. Seuret, H. Wendt, R. Leonarduzzi, S. Roux, and P. Abry, "Multivariate multifractal analysis," Applied and Computational Harmonic Analysis, vol. 46, no. 3, pp. 653-663, 2019.

[9] H. Wendt, R. Leonarduzzi, P. Abry, S. Roux, S. Jaffard, and S. Seuret, "Assessing cross-dependencies using bivariate multifractal analysis," in IEEE Int. Conf. Acoust., Speech, and Signal Proces. (ICASSP), Calgary, Canada, April 2018.

[10] R. Leonarduzzi, P. Abry, S. G. Roux, H. Wendt, S. Jaffard, and S. Seuret, "Multifractal characterization for bivariate data," in Proc. European Signal Processing Conference (EUSIPCO), Rome, Italy, September 2018.

[11] H. Wendt, P. Abry, and S. Jaffard, "Bootstrap for empirical multifractal analysis," IEEE Signal Proc. Mag., vol. 24, no. 4, pp. 38-48, 2007.

[12] M. Ben Slimane, "Baire typical results for mixed hölder spectra on product of continuous besov or oscillation spaces," Mediterr. J. Math., vol. 13, pp. 1513-1533, 2016.

[13] P. Mattila, Geometry of Sets and Measures in Euclidean Spaces: Fractals and Rectifiability. Cambridge Studies in Advanced Mathematics, 1999.

[14] K. Falconer, "Sets with large intersection properties," J. London Math. Soc. (2), vol. 49, no. 2, pp. 267-280, 1994.

[15] A. Durand, "Sets with large intersection and ubiquity," Mathematical Proceedings of the Cambridge Philosophical Society, vol. 144, no. 1, pp. 119-144, 2008.

[16] J. M. Aubry and S. Jaffard, "Random wavelet series," Comm. Math. Phys., vol. 227, no. 3, pp. 483-514, 2002.

[17] S. Jaffard, "The multifractal nature of Lévy processes," Probability Theory and Related Fields, vol. 114, no. 2, pp. 207-227, 1999.

[18] A. Durand, "Singularity sets of Lévy processes," Probab. Theory Relat. Fields, vol. 143, pp. 517-544, 2009. 